\title{
Analysis of machining characteristics in drilling of GFRP composite with application of fuzzy logic approach
}

\author{
B.C. Routara", A.K. Sahoo, Akshay K. Rout, A. K. Parida and J. R. Behera
}

School of Mechanical Engineering, KIIT University, Bhubaneswar, India

\begin{tabular}{l}
\hline C H R O N I C L E \\
\hline Article history: \\
Received June 12013 \\
Received in revised format \\
July 152013 \\
Accepted August 12013 \\
Available online \\
August 3 2013 \\
\hline Keywords: \\
Grey Relational analysis \\
Taguchi Method \\
ANOVA \\
Surface Roughness \\
\hline
\end{tabular}
A B S T R A C T

\begin{abstract}
This paper discusses the application of the Taguchi method to optimize the machining parameters for machining of GFRP composite in drilling for individual responses such as thrust force and delamination factor. Moreover, a multi-response performance characteristic is used for optimization of process parameters with application of grey relational analysis. An orthogonal array (L9), grey relational generation, grey relational coefficient and grey - fuzzy grade obtained from the grey relational analysis applied as performance index to solve the optimization problem of drilling parameters for GFRP composite. Taguchi orthogonal array, the signal-to-noise ratio, and the analysis of variance are used to investigate the optimal levels of cutting parameters. The confirmation tests are conducted to verify the results and it is observed that grey-fuzzy approach is efficient in determining the optimal cutting parameters.
\end{abstract}

\section{Introduction}

Composite materials are engineering materials made from two or more constituent materials that remain separate and distinct on a macroscopic level while forming a single component. Fiber reinforced composites are composed of fibers and a matrix. Fibers are the reinforcement and the main source of strength while matrix glues all the fibers together in shape and transfers stresses between the reinforcing fibers. The fibers carry the loads along their longitudinal directions. Filler might be added to smooth the manufacturing process, improve the mechanical properties to the composites, and / or reduce the product cost. The glass fiber-reinforced plastic (GFRP) composites are widely used in many of engineering applications. Machining of such composites is highly necessary for the concern of precision and accuracy. Drilling is the one of machining operation where delamination or damage of composites occurs frequently. Thrust force is the most concern for delaminarion of composites. Latha and Senthilkumar (2009a, 2009b, 2010) analyzed the thrust force and surface roughness in drilling operation. Fuzzy rule-based model has been developed to predict the thrust force. Response surface 
model has been developed and comparison between fuzzy based model and response surface model has been carried out. Singh et al. (2009) predicts the effect of process parameter on thrust force and torque by using the Fuzzy logic. Unlike drilling, the turning operation has been conducted on GFRP. Rajasekaran et al. (2011) conducted experiment on CFRP for surface roughness during turning. Guo et al. (2012) studied the surface roughness on machining of GFRP composites. According to their investigation, higher the cutting speed, the rougher and the more damaged the machined surface. Surface roughness has received serious attentions, as Surface finish is an important parameter in machining process. When GFRP composites are machined, it is clearly seen that the fibers are cut across and along their lay direction, leaving deformed projecting and partially disclosed fibers on the machined surface (Palanikumar et al., 2006; Feng et al., 2002; Sahoo \& Sahoo, 2011; Kumar et al., 2013). However, in various machining operations like EDM (Lin \& Lin, 2005), milling and turning operations grey relational relation is used. Palanikumar (2010) and Palanikumar et al. (2012) used the grey relational analysis to predict the efficient drilling process parameter for surface roughness and delamination. However, very few researchers have used the Grey-Fuzzy technique for analyzing the influenced process parameter on drilling. Krishnamoorthy et al. (2012) used the Grey-fuzzy technique for finding out the effect of drilling process parameters on thrust force, torque, delamination and eccentricity of holes. It is found that feed rate is most influential process parameter in drilling of CFRP.

In the present work, a procedure has been taken to assess and optimize the process parameters in drilling operation to attain minimum thrust force and less delamination by incorporating: response table and response graph; analysis of variance (ANOVA) technique. This paper outlines that the application of grey relational analysis and fuzzy inference system coupled with Taguchi method to optimize quality of GFRP under drilling operation.

\section{Experimental details}

\subsection{Design of experiment}

Design of experiment has been used to reduce the experimentation cost and time. In this experimental scheme, possible combinations of levels are included according to Taguchi orthogonal array $\mathrm{L}_{9}(3 * * 2)$ where factors are 2 and runs are 9.

Table 1 shows the drilling parameters (influencing parameters) with its symbol and levels. Table 2 shows the Experimental layout using an $\mathrm{L}_{9}$ orthogonal array.

Table 1

Influencing parameters \& their levels

\begin{tabular}{ccc}
\hline Levels of parameters & Spindle speed (N, rpm) & Feed rate $(\mathrm{f}, \mathrm{mm} / \mathrm{min})$ \\
\hline 1 (Low) & 300 & 10 \\
2 (Medium) & 400 & 20 \\
\hline (High) & 500 & 30 \\
\hline
\end{tabular}

Table 2

Experimental layout using an $\mathrm{L}_{9}$ orthogonal array

\begin{tabular}{ccc}
\hline Expt. No & Spindle speed(A) & Feed rate(B) \\
\hline 1 & 1 & 2 \\
2 & 1 & 3 \\
3 & 1 & 1 \\
4 & 2 & 2 \\
5 & 2 & 3 \\
6 & 2 & 1 \\
7 & 3 & 2 \\
9
\end{tabular}




\subsection{Experimental set up}

The experiments are conducted on computer numerical control (CNC) machining centre. All the experiments were conducted on the CNC milling set up in the central tool room of the CIPET, PATIA, Bhubaneswar as shown in Fig. 1. The specification of the Machine is as follows: Spindle speed: 06000rpm, feed: $1-50 \mathrm{~mm} / \mathrm{min}$. The experiments are conducted for 3 different spindle speeds and 3 different feed rates based on Taguchi's orthogonal array. The control parameters are selected based on the literature study, availability of spindle speed and feed rate on the machine and the experience gained from the field of study.

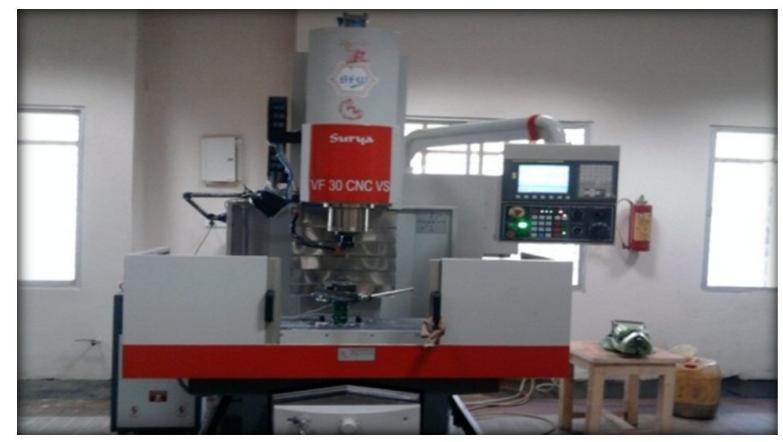

Fig. 1. Experimental set up for the drilling

\subsection{Work piece used}

Flat plates of Glass fibre reinforced polymer composite with $\mathrm{Al}_{2} \mathrm{O}_{3} /$ Fly ash as filler material is used as the specimen or work piece for drilling operation. The dimension of the each specimen is $20 \times 20 \times 5$ $\mathrm{mm}$. Brad and Spur drill having diameter of $8 \mathrm{~mm}$ is used for drilling operation.

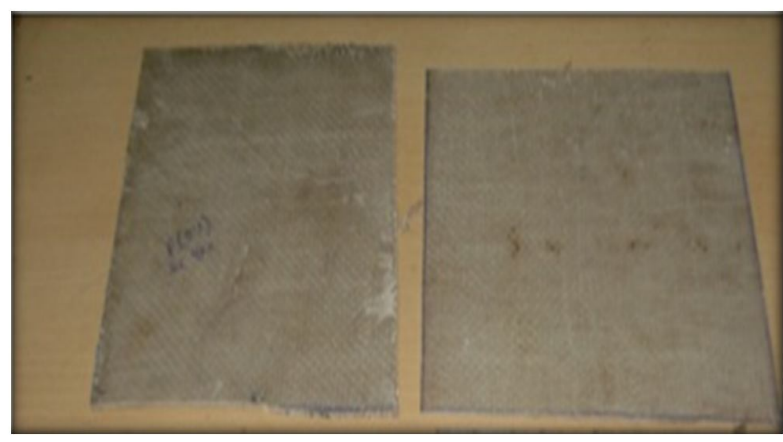

Fig. 2. Specimen for the drilling operation

\subsection{Response parameters}

The responses studied in the present work are Thrust force $\left(\mathrm{F}_{\mathrm{T}}\right)$ and Delamination factor $\left(\mathrm{F}_{\mathrm{d}}\right)$ :

Delamination in drilling is one of the important concerns for composite materials and is to be controlled. Delamination affects the hole surface and strength of the joined laminate. Delamination in drilling of composite occurs due to the thrust force developing during the operation.

The delamination factor can be expressed as:

$$
\mathbf{F}_{\mathbf{d}}=\mathbf{D}_{\max } / \mathbf{D}
$$

where $F_{d}$ is the delamination factor, $D_{\max }$ the maximum diameter of the damaged area, and $\mathrm{D}$ is the drill diameter as shown in Fig.3. 

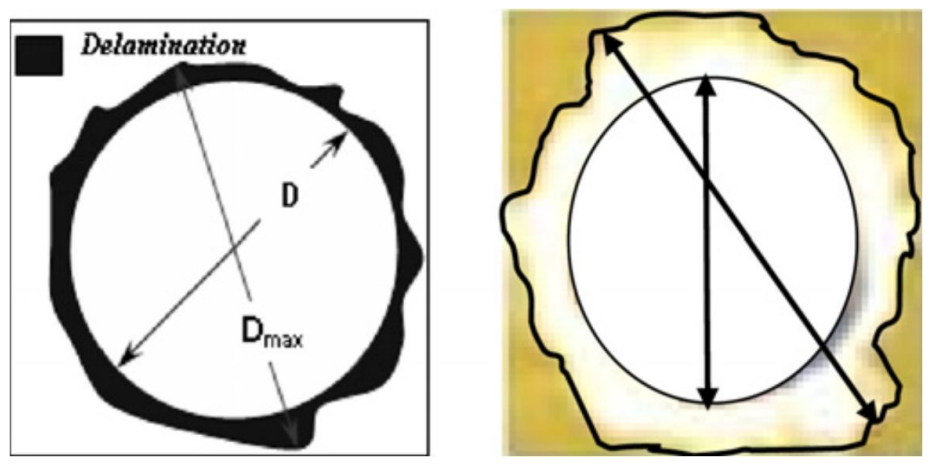

Fig. 3. Schematic diagram of delamination factor

In the present investigation delamination factor measured by a special attachment called Touch probe of the CNC machine centre at the central tool room of CIPET, BBSR. The photograph of the special attachment is shown in Fig 4.

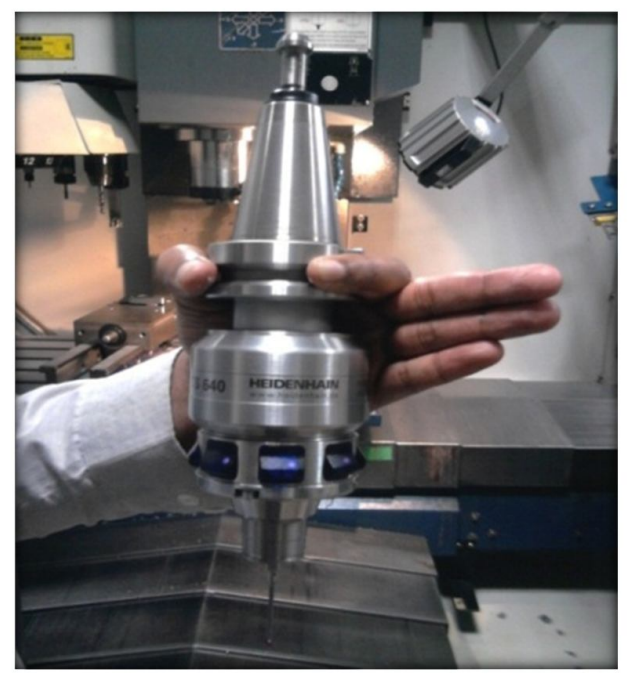

Fig. 4. Touch probe for measuring delamination

Thrust force in drilling operation has been measured by using Kistler piezoelectric type dynamometer at CIPET, BBSR. The experimental layout and experimental values of responses are reported on Table 3.

Table 3

Experimental values of $\mathrm{F}_{\mathrm{T}} \& \mathrm{~F}_{\mathrm{d}}$

\begin{tabular}{ccccccc}
\hline Expt. no & \multicolumn{2}{l}{ Spindle speed(rpm) } & \multicolumn{2}{l}{ Feed rate $(\mathrm{mm} / \mathrm{min})$} & $\begin{array}{c}\text { Thrust force } \\
(\text { Kgf })\end{array}$ & $\begin{array}{c}\text { Delamination } \\
\text { factor }\end{array}$ \\
& Coded & Actual & Coded & Actual & & \\
\hline 1 & 1 & 300 & 1 & 10 & 293 & 1.038 \\
2 & 1 & 300 & 2 & 20 & 296 & 1.020 \\
3 & 1 & 300 & 3 & 30 & 291 & 1.024 \\
4 & 2 & 400 & 1 & 10 & 286 & 1.004 \\
5 & 2 & 400 & 2 & 20 & 286 & 1.033 \\
6 & 2 & 400 & 3 & 30 & 288 & 1.034 \\
7 & 3 & 500 & 1 & 10 & 282 & 1.049 \\
\hline 8 & 3 & 500 & 2 & 20 & 287 & 1.054 \\
\hline 9 & 3 & 500 & 3 & 30 & 287 & \\
\hline
\end{tabular}




\section{Determination of optimum process parameter}

The grey relational analysis and Fuzzy-Taguchi approach have been used for this work for the selection of cutting parameters, optimization and its influence. The implementation of this method and results are discussed in following steps:

Step 1:

Grey relational analysis (Lin \& Lin, 2005; Palanikumar et al., 2012) is carried out following the steps outlined in previous section. It has already been mentioned earlier that thrust force and delamination factor need to be as smaller as possible. In grey relational analysis, the normalized data processing for thrust force and delamination factor corresponding to lower-the-better criterion (LB) can be expressed as

$$
x_{i}(k)=\frac{\max y_{i}(k)-y_{i}(k)}{\max y_{i}(k)-\min y_{i}(k)},
$$

where $x_{i}(k)$ is the value after the grey relational generation, $\min y_{i}(k)$ is the smallest value of $y_{i}(k)$ for the $k_{\text {th }}$ response, and $\max y_{i}(k)$ is the largest value of $y_{i}(k)$ for the $k_{\text {th }}$ response. An ideal sequence is $x_{0}(k)$ $(k=1 \& 2)$ for thrust force and delamination factor respectively). Basically, the larger normalized results correspond to the better performance and the best-normalized results should be equal to one shown in the Table 4.

Next, the grey relational coefficient is calculated to express the relationship between the ideal (best) and actual normalized experimental results.

The grey relational coefficient $\xi_{i}(k)$ can be calculated as

$$
\xi_{i}(k)=\frac{\Delta_{\min }+\psi \Delta_{\max }}{\Delta_{o i}(k)+\psi \Delta_{\max }},
$$

where $\Delta_{0 i}=\| x_{0}(k)-x_{i}\left(k \|=\right.$ difference of the absolute value between $x_{0}(k)$ and $x_{i}(k), \Delta_{\min }$ and $\Delta_{\max }$ are respectively the minimum and maximum values of the absolute differences of all comparing sequences. $\psi$ is a distinguishing coefficient, $0 \leq \psi \leq 1$, the purpose of which is to weaken the effect of $\Delta_{\max }$ when it gets too big and thus enlarges the difference significance of the relational coefficient. In the present case, $\psi=0.5$ is used.

\section{Table 4}

Grey relational generations

\begin{tabular}{ccc}
\hline $\begin{array}{c}\text { Exp. No } \\
\text { Ideal Sequence }\end{array}$ & $\mathrm{F}_{\mathrm{T}}$ & $\mathrm{F}_{\mathrm{d}}$ \\
\hline 1 & 1.000000 & 1.000000 \\
\hline 2 & 0.214286 & 1.000000 \\
3 & 0.000000 & 0.709524 \\
4 & 0.357143 & 0.609524 \\
5 & 0.714286 & 0.371429 \\
6 & 0.714286 & 0.533333 \\
7 & 0.571429 & 0.409524 \\
8 & 1.000000 & 0.390476 \\
9 & 0.642857 & 0.019048 \\
\hline
\end{tabular}

The grey relational coefficient of responses such as thrust force and delamination factor are calculated based on the Eq. (2). The obtained results are represented in Table 5. Most of the researchers applied Taguchi method to optimize the process parameters by taking overall grey relational grade (Palanikumar et al., 2012). However, there may arise some uncertainty due to co-relation of responses 
and response weight assignment. In order to overcome uncertainty fuzzy inference system has been used in this work.

\section{Table 5}

Grey relational coefficients of $\mathrm{F}_{\mathrm{T}}$ and $\mathrm{F}_{\mathrm{d}}$

\begin{tabular}{ccc}
\hline Exp. $\mathbf{N o}$ & $\mathbf{F}_{\mathbf{T}}$ & $\mathbf{F}_{\mathbf{d}}$ \\
\hline 1 & 0.214286 & 1.000000 \\
2 & 0.000000 & 0.709524 \\
3 & 0.357143 & 0.609524 \\
4 & 0.714286 & 0.371429 \\
5 & 0.714286 & 0.533333 \\
6 & 0.571429 & 0.409524 \\
7 & 1.000000 & 0.390476 \\
8 & 0.642857 & 0.019048 \\
9 & 0.642857 & 0.000000 \\
\hline
\end{tabular}

\section{Fuzzy inference system}

In the study, the fuzzifier firstly uses membership functions to convert the crisp inputs into fuzzy sets, and then the inference engine performs a fuzzy reasoning on fuzzy rules to generate fuzzy values, then the defuzzifier converts these values into the crisp outputs. Fuzzy values are determined by the membership functions that define the degree of membership of an object. Based on the fuzzy rules, the Mamdani implication method has been used for the fuzzy inference reasoning in this study (Fig. 5).

\section{Fuzzy rule:}

Let, $i=1,2,3, \ldots \ldots \ldots \ldots . . ., N$,

$R_{i}$ :If $x_{1}$ is $A_{i 1}, x_{2}$ is $A_{i 2}, \ldots$, and $x_{m}$ is, $A_{i m}$ then $y_{i}$ is $C_{i}$, where $N$ is the total number of fuzzy rules, $x_{1}, x_{2}, \cdots, x_{m}$ are the input variables i.e., $x_{j}$ ( let $j=1,2,3 \ldots . m$,), $y_{i}$ are the output variables, and $A_{i j}$ and $C_{i}$ are fuzzy sets defined by corresponding membership function $\mu_{A_{i j}}\left(x_{j}\right)$ and $\mu_{C_{i}}\left(y_{i}\right)$, respectively. Based on Mamdani implication, the aggregated output for the M rules is given by Eq. (3):

$\left.\mu c_{i}\left(y_{i}\right)=\max \left\{\min \mid \mu_{A i 1}\left(x_{1}\right), \mu_{A i 2}\left(x_{2}\right), \ldots \ldots \ldots \ldots \mu_{A_{i j}}\left(x_{m}\right)\right]\right\}$.

Using defuzzification method, fuzzy values are combined to form single output value by using centre of gravity formula. The formula for centroid of output $\hat{y}_{i}$ is given by Eq. (4):

$$
\hat{y}_{i}=\frac{\int y_{i} \mu_{c i}\left(y_{i}\right) d y}{\int \mu_{c i}\left(y_{i}\right) d y}
$$

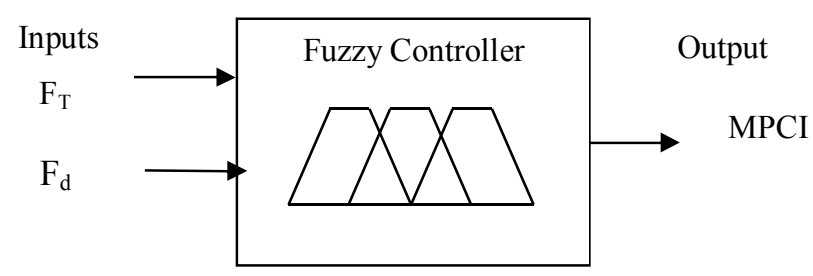

Fig.5. Structure of the two-input-one-output fuzzy logic unit.

Step 2: The Individual grey relational coefficients of $F_{T} \& F_{d}$ are taken as input responses of fuzzy inference system and only output is MPCI. In this work, six trapezoidal individual membership functions are designed for the three input responses as shown in Figure 6 (a \& b). Fifteen trapezoidal membership functions are assigned to the only output MPCI (Fig. 7). 


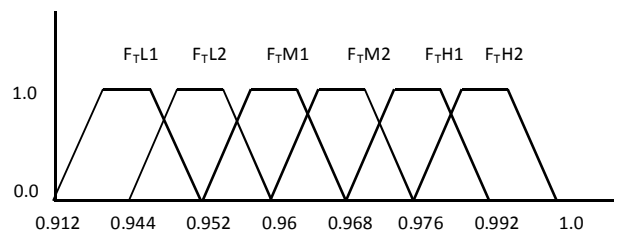

(a) Figure of membership functions for $\mathrm{F}_{\mathrm{T}}$

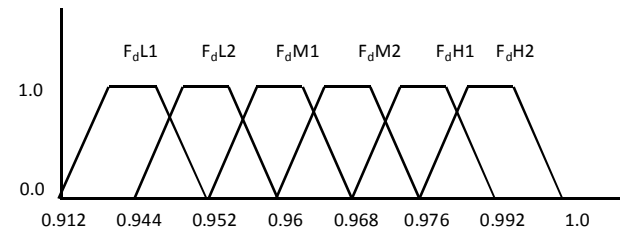

(b) Figure of membership functions for $F_{d}$

Fig. 6. Membership functions for responses

As shown in Fig. 6, there are six membership functions for input parameters: For $\mathrm{F}_{\mathrm{T}}$ low (L1, L2), medium (M1, M2), high (H1, H2). Similarly, for other input parameter $\mathrm{F}_{\mathrm{d}}$ the membership functions is given. For output parameter, MPCI fifteen membership functions are taken as follows: low (L1, L2, L3, L4, L5) medium (M1, M2, M3, M4, M5) and high (H1, H2, H3, H4, H5).

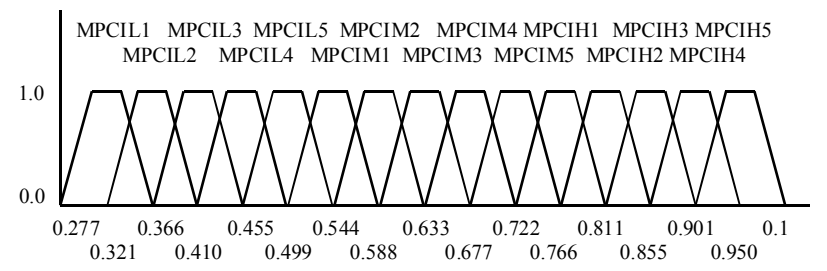

Fig. 7. Membership functions of output MPCIs

After the two input variables are fuzzified into the appropriate linguistic values, applying the fuzzy rules along with Mamdani inference, the fuzzy linguistic values and their membership values for the output MPCI can be obtained. Finally, defuzzification can be done by the centroid method using in Eq. (4) to convert fuzzy inference output $\left(\mu_{c i}\right)$ to crisp value $\left(\hat{y}_{i}\right)$ i.e. MPCIs. Table 6 shows the result of MPCI values corresponding number of experiments by using MATLAB.2009b generated fuzzy logic unit (Fig. 7). Larger the value of MPCI indicates better the quality and productivity performance. Based on aforesaid, quality performance characteristics is the best at larger MPCI but because of many parametric combinations it may not be optimal, therefore Taguchi method has been used to optimize the MPCIs.

Table 6

Results of MPCIs

\begin{tabular}{lccccccccc}
\hline Exp. No & 1 & 2 & 3 & 4 & 5 & 6 & 7 & 8 & 9 \\
\hline MPCI & 0.55010 & 0.79750 & 0.69280 & 0.67472 & 0.85726 & 0.83590 & 0.57390 & 0.73550 & 0.75620 \\
\hline
\end{tabular}

Step 3: Using Taguchi methodology, determine the values of $\mathrm{S} / \mathrm{N}$ ratio of corresponding all observed MPCIs which can select the optimal parameter setting that provides better productivity and quality of machined product. The $\mathrm{S} / \mathrm{N}$ ratios are calculated based on the larger-the-better criteria. Optimal machining parameter is selected based on maximum value of the $\mathrm{S} / \mathrm{N}$ ratio (Table 7). The effect of values of $\mathrm{S} / \mathrm{N}$ ratios on the process parameters is illustrated in main effect plot as shown in Fig. 8. It is clearly describes that, at Levels 2 corresponding $\mathrm{A}$, B have larger $\mathrm{S} / \mathrm{N}$ ratio respectively.

Table 7

Response for $\mathrm{S} / \mathrm{N}$ ratio

\begin{tabular}{ccc}
\hline Level & A & B \\
\hline 1 & -3.448 & -4.477 \\
2 & -2.104 & -1.990 \\
3 & -3.306 & -2.391 \\
Delta & 1.344 & 2.487 \\
Rank & 2 & 1 \\
\hline
\end{tabular}


Based on above discussion, the optimal machining parameter combination is A2B2 i.e. the speed at level $2(\mathrm{~A}=400 \mathrm{rpm})$, feed rate at level $2(\mathrm{~B}=20 \mathrm{~mm} / \mathrm{min})$ Feed rate having most significant effect on the thrust force and delamination factor.

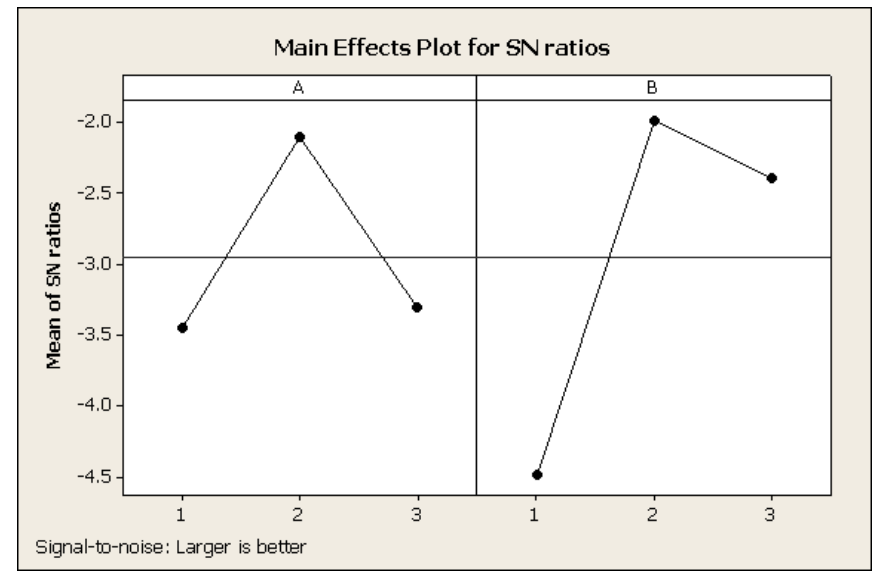

Fig. 8. Main effect plot for $\mathrm{S} / \mathrm{N}$ ratio

\section{Analysis of variance}

The purpose of the analysis of variance (ANOVA) is to investigate which machining parameters significantly affect the performance characteristic

Table 8

ANOVA for Grey-Fuzzy grade

\begin{tabular}{cccccc}
\hline Source & DF & SS & MS & F & P \\
\hline A & 2 & 0.022139 & 0.01107 & 10.27 & 0.027 \\
B & 2 & 0.066377 & 0.033188 & 30.79 & 0.004 \\
Error & 4 & 0.004311 & 0.001078 & & \\
Total & 8 & 0.092827 & & & \\
\hline
\end{tabular}

This is accomplished by separating the total variability of the grey-fuzzy reasoning grades, which is measured by the sum of the squared deviations from the total mean of the grey-fuzzy reasoning grade, into contributions by each machining parameter and the error. In addition, the Fisher's $F$-test can also be used to determine which machining parameters have a significant effect on the performance characteristic. In this study, F- tabulated value is greater than the F-calculated for feed rate, which indicates that feed rate is most significant for multi-objective performance characteristics. In the ANOVA table, it shows P-value for feed rate is very much less than 0.05.It proves that feed rate is significant with $95 \%$ confidence level.

\section{Confirmation test}

After the optimal level of machining parameters has been identified, a verification test needs to be carried out in order to check the accuracy of analysis. The estimated grey-fuzzy grade, $\hat{\gamma}$, using the optimal level of the process parameters can be calculated as:

$$
\hat{\gamma}=\gamma_{m}+\sum_{i=1}^{o}\left(\bar{\gamma}_{i}-\gamma_{m}\right)
$$


where $\gamma_{m}$ is the total mean grey-fuzzy reasoning grade, $\bar{\gamma}_{i}$ is the mean grey reasoning grade at the optimal level, and $o$ is the number of the main design parameters that significantly affect the machining characteristics of drilling process. Table 9 shows the comparison of the estimated grey-fuzzy reasoning grade (calculated using Eq. (5)) with the actual grey-fuzzy reasoning grade obtained in experiment using the optimal cutting parameters. It may be noted that there is good agreement between the estimated value (0.7345) and experimental value (0.85726). The improvement of grey-fuzzy reasoning grade from initial parameter combination (A1B1) to the optimal parameter combination (A2B2) is 0.30716 .

Table 9

Confirmation test results

\begin{tabular}{cccc}
\hline & Initial machining parameter & \multicolumn{2}{c}{ Optimal machining parameter } \\
& & Prediction & Experimental \\
\hline Level & $\mathrm{A} 1 \mathrm{~B} 1$ & $\mathrm{~A} 2 \mathrm{~B} 2$ & $\mathrm{~A} 2 \mathrm{~B} 2$ \\
$\mathrm{~F}_{\mathrm{T}}$ & 293 & - & 286 \\
$\mathrm{~F}_{\mathrm{d}}$ & 1.038 & - & 1.004 \\
Grey-Fuzzy Grade & 0.55010 & 0.7345 & 0.85726 \\
\hline
\end{tabular}

Improvement $=0.30716$

\section{Conclusion}

This paper outlines that the application of grey relational analysis and fuzzy inference system coupled with Taguchi method to optimize quality of GFRP under drilling operation. The optimization of machining parameters is carried out with minimum number of test conditions by using orthogonal array. Fuzzyfication technique helps to avoid the vagueness in the results and provide the best cutting conditions. Based on experimental results, the following conclusions are:

(1) The parameter combination second level of spindle speed and second level of feed rate are evaluated for optimal parameters for machining of GFRP on drilling operation.

(2) Multi-response objectives are optimized in very simple, effective and efficient manner.

(3) Feed rate has the most significant parameter with consideration of multiple-objective characteristics.

The use of the orthogonal array with grey-fuzzy logic to optimize the drilling process with the multiple performance characteristics has been reported here. A grey relational analysis of the experimental results of machining responses such as thrust force and delamination factor can convert optimization of a single performance characteristic. As a result, optimization of the complicated multiple performance characteristics can be greatly simplified through this approach.

\section{References}

Guo, D.M., Wen, Q., Gao, H., \& Bao, Y.J (2012). Prediction of the cutting forces generated in the drilling of carbon-fibre-reinforced plastic composites using a twist drill. Proceedings of the Institution of Mechanical Engineers, Part B: Journal of Engineering Manufacture, 226(1), 28-42.

Krishnamoorthy, A., Rajendra Boopathy, S., Palanikumar, K., \& Paulo Davim, J. (2012).Application of grey fuzzy logic for the optimization of drilling parameters for CFRP composites with multiple performance characteristics. Measurement, 45(5), 1286-1296.

Kumar, R., Sahoo, A., Satyanarayana, K., \& Rao, G. (2013). Some studies on cutting force and temperature in machining Ti-6Al-4V alloy using regression analysis and ANOVA. International Journal of Industrial Engineering Computations, 4(3), 427-436. 
Latha, B., \& Senthilkumar, V. S. (2009). Analysis of thrust force in drilling glass fiber-reinforced plastic composites using fuzzy logic. Materials and Manufacturing Processes, 24(4), 509-516.

Latha, B., \& Senthilkumar, V. S. (2010). Modeling and analysis of surface roughness parameters in drilling GFRP composites using fuzzy logic. Materials and Manufacturing Processes, 25(8), 817 827.

Latha, B., \& Senthilkumar, V. S. (2009). Fuzzy rule based modeling of drilling parameters for delamination in drilling GFRP composites. Journal of Reinforced Plastics and Composites, 28(8), 951-964.

Lin, J.L., \& Lin, C.L. (2005). The use of grey-fuzzy logic for the optimization of the manufacturing process. Journal of Materials Processing Technology, 160, 9-14.

Feng, C., X., Xian \& Feng, W. (2002). Development of empirical models for surface roughness prediction in finish turning. International Journal of Advanced Manufacturing Technology, 20(5), 348-356.

Palanikumar, K., Karunamoorthy, L., \& Karthikeyan, R. (2006). Assessment of factors influencing surface roughness on the machining of glass fiber-reinforced polymer composites. Materials and Design, 27, 862-871.

Palanikumar, K. (2010). Modeling and analysis of delamination factor and surface roughness in drilling GFRP composites. Materials and Manufacturing Processes, 25(10), 1059-1067.

Palanikumar, K., Latha, B., Senthilkumar, V. S., \& Davim, J. P. (2012). Analysis on drilling of glass fiber-reinforced polymer (GFRP) composites using grey relational analysis. Materials and Manufacturing Processes, 27(3), 297-305.

Rajasekaran, T., Palanikumar, K., \& Vinayagam, B. K. (2011). Application of fuzzy logic for modeling surface roughness in turning CFRP composites using CBN tool. Production Engineering, 5(2), 191199.

Sahoo, A., \& Sahoo, B. (2011). Surface roughness model and parametric optimization in finish turning using coated carbide insert: Response surface methodology and Taguchi approach. International journal of industrial engineering computations, 2(4), 819-830

Singh, R. V. S., Latha, B., \& Senthilkumar, V. S. (2009). Modeling and analysis of thrust force and torque in drilling GFRP composites multi-facet drill using fuzzy logic. International Journal of Recent Trends in Engineering, 1(5), 66-70. 